\title{
TCEA1 Gene
}

National Cancer Institute

\section{Source}

National Cancer Institute. TCEA1 Gene. NCI Thesaurus. Code C97822.

This gene is involved in transcription. 